Poder de mercado y transmisión asimétrica de precios en Chile: el caso de la carne bovina y porcina

Ricardo Troncoso Sepúlveda 
Ricardo Troncoso Sepúlveda

\section{Poder de mercado y transmisión asimétrica de precios en Chile: el caso de la carne bovina y porcina}

Resumen: Este articulo examina el efecto del poder de mercado sobre la transmisión de precios productor-mayorista en las industrias de carne bovina y porcina chilena. Se utilizaron elasticidades conjeturales para representar el poder de mercado y se estimó un modelo de corrección de errores por umbral (TECM, por sus siglas en inglés) con tres regimenes para distinguir el comportamiento de ajuste de precios con relación al equilibrio de largo plazo. Los resultados indican que el poder de mercado tiene impacto significativo en el sector bovino cuando los precios se hallan en fase decreciente. En dicha fase, los mayoristas pueden ejercer poder de mercado para ralentizar el ajuste de precios y conservar márgenes de ganancia mayores durante más tiempo. La hipótesis de simetría de largo plazo es rechazada en el sector bovino cuando se incorpora el poder de mercado y no rechazada cuando se ignora en la especificación del modelo, sugiriendo transmisión asimétrica entre los regimenes bajo y alto. Este resultado, refuerza la idea que el poder de mercado mayorista produce asimetrías en el ajuste de precios que son régimendependientes sólo en el sector bovino.

Palabras clave: transmisión de precios, poder de mercado, industrias bovina y porcina, umbral, modelo de corrección de errores.

Clasificación JEL: C32, Q13, L11.

\section{Market Power and Asymmetric Price Transmission in Chile: The case of bovine and porcine meat}

Abstract: This article examines the effect of market power on the price transmission from producer to wholesaler in the Chilean beef and pork. industries. Conjectural elasticities were used as a proxy for market power and a Threshold Error Correction Model (TECM) with three regimes was estimated to distinguish price adjustment behavior in relation to long-run equilibrium. The results indicate that market power has a significant impact in the bovine sector when prices are in a decreasing phase. In this phase, bovine wholesalers can exercise market power to slow down the price adjustment downwards and retain bigher profit margins for longer. The symmetry bypothesis in the long-run adjustment is rejected in cattle when market power is incorporated and does not reject when it is ignored in the model specification, suggesting asymmetric price transmission between the low and high regimes. This result reinforces the idea that wholesale market power produces asymmetries in the adjustment of prices that are regime-dependent only in the bovine sector.

Keywords: price transmission, market power, bovine and pork industries, threshold, error correction model.

\section{(cc) BY-NC-SA}

Este artículo y sus anexos se distribuyen por la revista Lecturas de Economía bajo los términos de la Licencia Creative Commons Atribución-NoComercial-CompartirIgual 4.0. https://creativecommons.org/licenses/by-nc-sa/4.0/ 


\section{Pouvoir de marché et transmission asymétrique des prix au Chili: le cas du marché de la viande de bœuf et de porc}

Résumé: Cet article examine l'effet du pouvoir de marché sur la transmission des prix de gros au producteur dans les industries chiliennes de la viande de bouf et de porc. Des élasticités conjecturales ont été utilisées pour représenter le pouvoir de marché, puis un modèle de correction d'erreur de seuil (TECM) a été estimé à partir de trois régimes, afin de distinguer le comportement d'ajustement des prix par rapport à l'équilibre de long terme. Les résultats indiquent que le pouvoir de marché a un impact significatif sur le secteur bovin lorsque les prix sont à la baisse. Dans cette phase descendante, les grossistes peuvent exercer un powvoir de marché pour ralentir l'ajustement des prix et avoir ainsi des marges de profits plus élevées à long terme. Dans la spécification du modèle, l'hypothèse de symétrie à long terme est rejetée pour le secteur bovin lorsque le pouvoir de marché est incorporé et non rejetée lorsqu'elle est ignorée. Tout ceci suggère l'existence d'une transmission asymétrique entre les régimes bas et haut. Ce résultat renforce l'idée selon laquelle le pouvoir du marché grossiste entraine des asymétries dans l'ajustement des prix qui ne dépendent du régime que dans le secteur bovin.

Mots clés: Transmission des prix, pouvoir de marché, filières bovine et porcine, seuil, modèle de correction d'erreurs.

Cómo citar / How to cite this item:

Troncoso-Sepúlveda, R. A. (2021). Poder de mercado y transmisión asimétrica de precios en Chile: el caso de la carne bovina y porcina. Lecturas de Economía, 95, 135-166.

https://doi.org/10.17533/udea.le.n95a343197 


\title{
Poder de mercado y transmisión asimétrica de precios en Chile: el caso de la carne bovina y porcina
}

\author{
Ricardo Troncoso Sepúlveda $\oplus^{\mathrm{a}}$
}

-Introducción. -I. Revisión de literatura. -II. Modelo y metodología. -III. Datos. -IV. Resultados empíricos. -Conclusiones. -Anexo. - Referencias.

Primera versión recibida el 27 de julio de 2020; versión final aceptada el 08 de febrero de 2021

\section{Introducción}

La carne, desde un punto de vista nutricional, posee proteínas, minerales y vitaminas de alta calidad y biodisponibilidad que hacen de ella un alimento altamente valorado. Mientras los países desarrollados no han experimentado variaciones considerables en el consumo de carne, según información de la FAO (FAO, 2019), en los países en desarrollo el consumo per cápita se ha duplicado desde 1980 debido, principalmente, al aumento de la población, al incremento de los ingresos y a cambios en las preferencias alimentarias. Sin embargo, en estos países los niveles de consumo presentan variaciones significativas dependiendo de la región: África registra el consumo más bajo (16 kilos) y Oceanía el mayor (33 kilos). Además, existen economías en desarrollo donde el consumo de carne no supera los 7 kilos per cápita anuales, como Bangladesh y Burundi con 4 kilos, Gambia con 6,4 kilos y Etiopía con 7 kilos, debido al bajo poder adquisitivo de la población; o la India, en donde el bajo consumo (3,7 kilos) se debe más bien a aspectos de tipo religioso. Indudablemente, el creciente mercado de la carne en estos países ha generado grandes oportunidades para los productores pecuarios, intermediarios y elaboradores. Durante 2018, la producción mundial de carne se estimó en 338,6 millones de toneladas experimentando un crecimiento del 1,5\% respecto de 2017 originado principalmente en Estados Unidos, la Unión Europea y Rusia,

a Ricardo Troncoso-Sepúlveda: Pontificia Universidad Católica de Chile, Chile. Dirección electrónica: rdtroncoso@uc.cl https://orcid.org/0000-0002-4573-8231 
Troncoso-Sepúlveda: Poder de mercado y transmisión asimétrica de precios en Chile...

pero compensado por un estancamiento de los mercados de China y Brasil (FAO, 2018).

En Chile, la producción de carnes se halla enfocada, principalmente, en tres industrias: avícola, porcina y bovina. La producción avícola, líder a nivel nacional con 765506 toneladas durante 2019, se caracteriza por estar concentrada geográfica e industrialmente. A nivel geográfico, un 59,8\% corresponde a las regiones de O’Higgins, Nuble, Biobío y La Araucanía, y un $28,2 \%$ a la región Metropolitana de Santiago. A nivel industrial, existen solo siete firmas que operan en el país y cada una está integrada en todos los procesos de la cadena, desde la crianza hasta la distribución, permitiendo asegurar un alto nivel de trazabilidad de los productos. El sector porcino, segundo en importancia con 529957 toneladas de carne en vara en 2019, concentra el 87,1\% de su producción en la región de O’Higgins y está caracterizado por ser intensivo, con niveles altos de tecnología, concentrado en pocos actores y con un importante foco hacia la exportación, destinando un $63 \%$ al mercado asiático: Japón $(27 \%)$, Corea del Sur $(23 \%)$ y China (17\%), según información de la Asociación Gremial de Productores de Cerdos de Chile (ASPROCER, 2019). La industria bovina, con 212,000 toneladas de carne en vara en 2019 distribuyó su producción, mayoritariamente, en las regiones de Los Ríos y Los Lagos (37,6\%), Metropolitana de Santiago (21,4\%) y La Araucanía (17,9\%). Es una industria focalizada en la comercialización y producción nacional y —al igual que los sectores avícola y porcino- se encuentra concentrada. Un 54\% del procesamiento total de carne bovina es controlado por las cuatro empresas de mataderos más grandes del país (Vargas \& Raddatz, 2004).

Las principales industrias de la carne en Chile presentan una característica común: están concentradas en pocos actores a lo largo de la cadena de suministro, lo que puede generar asimetrías en la transmisión de precios (APT, por sus siglas en inglés) desde un eslabón de la cadena productiva hacia otro, provocando pérdidas en las utilidades o en el bienestar de los consumidores (Yu \& Gould, 2019). En la literatura, la APT suele ser atribuida a dos causas: existencia de mercados no competitivos y costos de ajuste (Meyer \& von Cramon-Taubadel, 2005. Respecto de la primera, y especialmente en agricultura, ambos extremos de la cadena de comercialización (productores y 
consumidores) a menudo pueden sospechar que la competencia imperfecta permite a los intermediarios usar poder de mercado para manipular el ajuste de precios a su favor (Miller \& Hayenga, 2001; McCorriston, 2002; Meyer \& von Cramon-Taubadel, 2005). Si bien muchos estudios han señalado al poder de mercado como causa de la APT, muy pocos han estimado empíricamente su impacto, dando cabida a potenciales investigaciones, sobre todo en países en desarrollo.

El propósito principal de este estudio es estimar el efecto del poder de mercado mayorista sobre la transmisión de precios como respuesta a cambios en los precios al productor en la cadena de suministro de carne bovina y porcina chilena. Mediante el uso de elasticidades conjeturales y la especificación de un parámetro para representar la velocidad de ajuste de los precios mayoristas como función del poder de mercado, se estimó un modelo de corrección de errores por umbral (Tong, 1990; Chan, 1993; Blake \& Fomby, 1997; Enders \& Siklos, 2001) a fin de evaluar posibles asimetrías en el proceso de ajuste de precios e intentar responder a algunas interrogantes como las siguientes: ¿Existe poder de mercado mayorista en estas industrias?, ¿cuál es la influencia del poder de mercado en el comportamiento de ajuste de precios?, ¿qué industria presenta mayor poder de mercado y en cuál la APT es más aguda? y ¿cómo es la elasticidad precio-oferta de productores bovinos y porcinos? Una gran ventaja de los modelos de umbrales es que consideran las propiedades de las series de tiempo mediante su estructura basada en regímenes dando cabida a no linealidades en las relaciones de cointegración y a costos de transacción no observables en el proceso de transmisión de precios entre mercados.

La estructura del artículo consta de cinco apartados. En el primero, se muestra una revisión de los principales trabajos que han estudiado empíricamente el impacto del poder de mercado sobre la transmisión de precios. En el segundo apartado, se describe el modelo teórico y la metodología utilizada. En el tercero, se indican las fuentes de información utilizadas y se expone un análisis de las estadísticas descriptivas más relevantes. Finalmente, en los apartados cuarto y quinto, se detallan los resultados y las conclusiones del trabajo. 
Troncoso-Sepúlveda: Poder de mercado y transmisión asimétrica de precios en Chile...

\section{Revisión de literatura}

Desde la década del 50, con el trabajo seminal de Farrell (1952), se ha desarrollado una creciente literatura de métodos para estudiar la transmisión de precios y sus asimetrías, en productos agrícolas. El interés de algunos economistas por estudiar los mecanismos de transmisión responde a las implicaciones de la política en el mercado, la asignación de recursos y la distribución del bienestar cuando se presentan asimetrías. Autores como Geweke (2004), Meyer y von Cramon-Taubadel (2005) y Frey y Manera (2007) realizaron importantes trabajos de revisión de literatura en los cuales clasificaron los estudios por país y commodities, y advirtieron alta heterogeneidad en términos de modelos econométricos. Al respecto, los desarrollos incluyen aplicaciones a modelos autorregresivos de rezagos distributivos (Farrell, 1952; Wolffram, 1971; Houck, 1977), modelos de corrección de error con o sin linealidades en los mecanismos de transmisión de precios (Meyer, 2004; Cudjoe et al., 2010; Brosig et al., 2011; Götz et al., 2013; Tansuchat et al., 2016; Troncoso-Sepúlveda, 2019) y la implementación de modelos de cuantiles autorregresivos para estudiar desde una perspectiva integrada, elementos como la evolución de la distribución, existencia ciclos y su impacto en la APT agrícolas (Chavas \& Pan, 2019).

Sin embargo, y a pesar de la basta literatura existente, muy pocos trabajos han estimado empíricamente el efecto de factores contextuales sobre la APT agrícolas. Por ejemplo, Alfaro y Olivera (2009) analizaron el mercado de ganado vacuno para faena en Uruguay para determinar la influencia del poder de mercado sobre la transmisión de precios. Los hallazgos muestran que existe transmisión no perfecta entre el precio internacional y el precio al productor, lo que sugiere — según los autores - la necesidad de analizar más en profundidad la competencia en este mercado. Ceballos (2014) utilizando modelos de cointegración por umbral, estudió la presencia de asimetrías en la transmisión de precios en la cadena de valor de la carne bovina, porcina y de ave en Chile. Los resultados advierten la existencia de asimetrías positivas y negativas en los tres mercados y en distintos eslabones de la cadena de valor. Farías, et al. (2016), mediante un modelo de corrección del error, analizaron la transmisión de precios en la cadena de valor de la carne bovina 
en Chile. Sus hallazgos muestran que existe transmisión de precios desde el mercado internacional hacia el mercado nacional, y que también se produce transmisión entre los distintos eslabones de la cadena. Acharya et al. (2011) utilizaron un modelo mixto para estimar la transmisión de precios granjaminorista en el mercado estadounidense de fresas frescas. Los resultados sugieren dos regímenes de precios asociados con las temporadas de bajo y alto nivel de cosechas. Además, los cambios de precio se transmitieron completamente en el régimen de baja cosecha cuando el parámetro de poder de mercado fue cero, pero no en el régimen de alto cuando el parámetro fue positivo. Assefa et al. (2014) desarrollaron un modelo de oligopoliooligopsonio basado en las aproximaciones de Sexton y Zhang (2001) y Bunte y Peerlings (2003) para evaluar el grado de transmisión de precios en la cadena de suministro de papa holandesa. Mediante un experimento de simulación, mostraron que la transmisión puede llegar a ser imperfecta y asimétrica como consecuencia del poder oligopsónico minorista (detallista) en el sentido que una disminución del precio granja es parcialmente transmitida a los consumidores, mientras que un incremento se transmite completamente. Además, concluyen que el poder oligopsónico en agricultores puede incluso empeorar la transmisión de precios. Richards et al. (2014) explicaron porqué la tasa de transferencia de precios minorista — en la industria de cereales para el desayuno de Los Ángeles (Estados Unidos) — tiende a diferir dependiendo si los precios mayoristas aumentan o disminuyen. Hallaron que el poder de mercado, contrario al fenómeno rockets and feathers, provoca que los precios minoristas caigan rápidamente y aumenten lentamente, mientras que el efecto de consumer search es el opuesto y permite explicar los patrones de ajuste asimétrico más frecuentemente observados en los datos.

Assefa et al. (2017) investigaron la relación entre la volatilidad de precios y el poder de mercado en la cadena de suministro de carne fresca de cerdo en Alemania. Mediante un modelo teórico basado en los trabajos de Sexton \& Zhang (2001) y Verreth et al. (2015) y la aplicación de un modelo de corrección del vector de error (VECM, por sus siglas en inglés) y una regresión por mínimos cuadrados ordinarios (MCO) para analizar la transmisión de precios y volatilidad, mostraron que el poder de mercado de minoristas limita ambos tipos de transmisiones. Liu et al. (2019) examinaron 
el efecto heterogéneo de productos de etiqueta privada y de marca sobre la transmisión asimétrica de precios en el mercado de leche fluida, usando un modelo de corrección de error asimétrico de umbral. Los resultados indican que el ajuste de precios minoristas hacia arriba es más rápido que el ajuste hacia abajo en ambos tipos de productos. Además, hallaron que el poder de mercado minorista y las regulaciones estatales de precios contribuyen a la heterogeneidad de la transmisión asimétrica de precios. A mayor poder de mercado, los precios de la leche de etiqueta privada aumentan más rápido cuando los precios minoristas están aumentando y caen más lentamente cuando se reducen. La existencia de regulaciones de precios ralentiza la velocidad de ajuste al equilibrio de largo plazo de precios de etiquetas privadas, independientemente de si el precio de venta es bajo o alto.

Yu y Gould (2019) analizaron el impacto de la competitividad de mercado sobre el grado de transmisión asimétrica de precios e implicaciones asociadas al bienestar. Mediante la estimación de un sistema casi ideal de demanda, un modelo de rezagos distribuidos y un modelo de corrección de errores para productos lácteos líquidos en 18 áreas metropolitanas de EE. UU., demostraron que una menor competencia produce mayor asimetría y que se traslada en un impacto mayor sobre el bienestar de los consumidores. Además, hallaron que la pérdida de bienestar bajo transmisión asimétrica de precios es más grande cuando se mide como porcentaje del gasto en productos lácteos. Surathkal y Chung (2019) estimaron el efecto del manejo de inventarios y poder de mercado de los empacadores sobre su comportamiento de ajuste de precios en respuesta a cambios en los precios al productor y minorista en la cadena de suministro de carne bovina de EE. UU. Los resultados muestran que, en la fase decreciente, los inventarios aceleran el proceso de ajuste de precios mientras que el poder de mercado ralentiza su disminución. Además, no se halló evidencia estadística contra el ajuste asimétrico de precios cuando se incorporan ambos factores contextuales en el análisis.

Este artículo está estrechamente relacionado con la literatura existente y, es el primer intento que analiza empíricamente la influencia del poder de mercado sobre la transmisión de precios y utilizar un marco teórico para recuperar elasticidades precio-oferta en las cadenas de suministro de carne bovina y porcina en Chile. 


\section{Modelo y metodología}

\section{A. Threshold error correction model (TECM)}

Para estudiar el comportamiento de ajuste de precios, se usó un procedimiento secuencial y una especificación TECM similar a la propuesta por Radchenko (2005) y Lewis (2011) para capturar asimetrías intra e inter-regímenes. En la primera etapa del procedimiento, se estimaron los parámetros de umbral. En la segunda etapa, se estimaron las asimetrías y los términos de transmisión de precios de largo y corto plazo basados en los umbrales obtenidos en la etapa previa.

En el largo plazo, si los precios a mayorista $\left(P_{w, t}\right)$, productor $\left(P_{p, t}\right)$, salarios $\left(P_{s, t}\right)$ y electricidad $\left(P_{e, t}\right)$ están cointegrados, la relación puede ser especificada como: ${ }^{1}$

$$
P_{w, t}=a_{0}+a_{1} P_{p, t}+a_{2} P_{s, t}+a_{3} P_{e, t}+\varepsilon_{t},
$$

donde $a_{0}, a_{1}, a_{2}$ y $a_{3}$ son parámetros a estimar, y $\varepsilon_{t}$ es el término de error normalmente distribuido que representa la desviación desde la relación de equilibrio entre los precios. Para probar la presencia de cointegración no lineal, se probó la hipótesis nula $\left(H_{0}\right)$ de proceso lineal de raíz unitaria para $\varepsilon_{t}\left(E C T_{t}\right)$ contra la hipótesis alternativa $\left(H_{A}\right)$ de estacionariedad no lineal en tres regímenes de la forma MTAR (momentum threshold autoregressive, por sus siglas en ingles), siguiendo la prueba univariada de Bec et al. (2004) basado en tres estadísticos diferentes: sup-LR, sup-LM y sup-Wald. Posteriormente, a fin de asegurar que el ajuste hacia el equilibrio es mejor representado por un modelo con tres regímenes, se utilizó la prueba de Hansen para contrastar la $H_{0}$ de un régimen con $H_{A}$ de tres regímenes ${ }^{2}$. Luego, el MTAR con tres regímenes es especificado como:

1 Bajo las condiciones definidas en Engle y Granger (1987).

2 Los modelos con tres regímenes (dos umbrales) son más flexibles porque incluyen una banda neutral o régimen medio que facilita la posibilidad de asimetría en el ajuste de precios (Meyer \& von Cramon-Taubadel, 2004). 
Troncoso-Sepúlveda: Poder de mercado y transmisión asimétrica de precios en Chile...

$$
\Delta E C T_{t}=\left\{\begin{array}{c}
\eta_{0}^{B}+\eta_{1}^{B} E C T_{t-1}+\sum_{p=1}^{p} \eta_{2, p}^{B} \Delta E C T_{t-p}+e_{t}^{B} \\
\text { si } \Delta E C T_{t-1} \leq \tau_{1} \\
\eta_{0}^{M}+\eta_{1}^{M} E C T_{t-1}+\sum_{p=1}^{p} \eta_{1, p}^{M} \Delta E C T_{t-p}+e_{t}^{M} \\
\text { si } \tau_{1}<\Delta E C T_{t-1} \leq \tau_{2} \\
\eta_{0}^{A}+\eta_{1}^{4} E C T_{t-1}+\sum_{p=1}^{p} \eta_{2, p}^{4} \Delta E C T_{t-p}+e_{t}^{A} \\
\text { si } \Delta E C T_{t-1}>\tau_{2}
\end{array}\right.
$$

Siendo $E C T_{t}$ el término de corrección de error y $\eta$ parámetros a ser estimados. Los términos de umbral, $\tau_{1}$ y $\tau_{2}$, fueron estimados mediante un procedimiento de búsqueda de grillas en donde la variable umbral fue ordenada ascendentemente, el 10\% de los primeros y últimos valores fueron excluidos para asegurar un mínimo de observaciones en cada régimen y evitar datos atípicos, y posteriormente la SSR fue estimada para cada valor, seleccionando como estimador aquel que minimizara la SSR.

Una vez obtenido el valor de los umbrales, el proceso AR de (2) se extendió a un TECM para capturar la dinámica de corto y largo plazo y el impacto del poder de mercado sobre la transmisión de precios productormayorista. Así, el TECM propuesto puede ser especificado como:

$$
\Delta P_{v, t}=\left\{\begin{array}{l}
\gamma_{0}+\Theta_{t}^{B}\left(M P_{t}\right) E C T_{t-1}+\sum_{k=1}^{k} \gamma_{1, k}^{B} \Delta P_{w, t-k}^{B} \\
+\sum_{k=0}^{k} \beta_{1, k}^{B} \Delta P_{p, t-k}^{+B}+\sum_{k=0}^{k} \beta_{2, k}^{B} \Delta P_{p, t-k}^{-B} \\
+\gamma_{2}^{B} \Delta P_{s}^{B}+\gamma_{3}^{B} \Delta P_{e}^{B}+u_{t}^{B}, \operatorname{si} E C T_{t-1} \leq \tau_{1} \\
\gamma_{0}+\Theta_{t}^{M}\left(M P_{t}\right) E C T_{t-1}+\sum_{k=1}^{k} \gamma_{1, k}^{M} \Delta P_{w, t-k}^{M} \\
+\sum_{k=0}^{k} \beta_{1, k}^{M} \Delta P_{p, t-k}^{+M}+\sum_{k=0}^{k} \beta_{2, k}^{M} \Delta P_{p, t-k}^{-M} \\
+\gamma_{2}^{M} \Delta P_{s}^{M}+\gamma_{3}^{M} \Delta P_{e}^{M}+u_{t}^{M}, \operatorname{si} \tau_{1}<E C T_{t-1} \leq \tau_{2} \\
\gamma_{0}+\Theta_{t}^{A}\left(M P_{t}\right) E C T_{t-1}+\sum_{k=1}^{k} \gamma_{1, k}^{A} \Delta P_{w, t-k}^{A} \\
+\sum_{k=0}^{k} \beta_{1, k}^{A} \Delta P_{p, t-k}^{+A}+\sum_{k=0}^{k} \beta_{2, k}^{A} \Delta P_{p, t-k}^{-4} \\
+\gamma_{2}^{A} \Delta P_{s}^{A}+\gamma_{3}^{A} \Delta P_{e}^{A}+u_{t}^{A}, \mathrm{si} E C T_{t-1}>\tau_{2},
\end{array}\right.
$$

donde $\Delta P_{w, t}$ y $\Delta P_{p, t}$ son las primeras diferencias de los precios a mayorista y productor en el período $t ; \gamma_{0}, \gamma_{1, k}, \gamma_{2}, \gamma_{3}, \beta_{1, k}$ y $\beta_{2, k}$ parámetros a ser estimados con $\gamma_{s}$ y $\beta_{s}$ representando eventuales asimetrías de corto plazo; y 
$u_{t}$ un término de error $\ddot{i i d . ~} \Theta_{t}\left(M P_{t}\right)$ representa la velocidad de ajuste hacia el equilibrio de largo plazo en cada régimen, como una función del poder de mercado $\left(M P_{t}\right)$. Específicamente, $\Theta_{t}\left(M P_{t}\right)$ toma una forma lineal como:

$$
\begin{aligned}
& \Theta_{t}^{B}=\theta_{0}^{B}+\theta_{1}^{B} M P_{t} \\
& \Theta_{t}^{M}=\theta_{0}^{M}+\theta_{1}^{\mathrm{M}} M P_{t} \\
& \Theta_{t}^{A}=\theta_{0}^{A}+\theta_{1}^{A} M P_{t} .
\end{aligned}
$$

$M P_{t}$ se representó mediante la elasticidad conjetural tiempo-variante mayorista/productor (Surathkal \& Chung, 2019) obtenida según el modelo de ecuaciones simultáneas propuesto por Muth y Wohlgenant (1999) que, basado en la estimación por 3SLS de funciones de demanda y oferta, permite generar una variable proxy para la concentración de mercado en una industria con limitada disponibilidad de datos (ver Anexo).

La asimetría general, o ajuste asimétrico de largo plazo, se testeó con la hipótesis: $H_{A}: \Theta_{t}^{B}=\Theta_{t}^{A}$ versus $H_{A}: \Theta_{t}^{B} \neq \Theta_{t}^{A}$. Ambos coeficientes incorporan el rol del poder de mercado en la transmisión de precios desde productor a mayorista a través de los $\theta_{1}$, términos de interacción entre elasticidad conjetural y variables de corrección de errores. Las elasticidades conjeturales más altas implican mayor poder de negociación; por lo tanto, un coeficiente $\theta_{1}$ grande y estadísticamente significativo conlleva a un fuerte efecto de poder de mercado en el ajuste de precios mayoristas. La diferencia estadística del poder de mercado entre los regímenes bajo y alto se analizó probando $H_{A}: \Theta_{1}^{B}=\Theta_{1}^{A}$ respecto de $H_{A}: \Theta_{1}^{B} \neq \Theta_{1}^{A}$. Los efectos de asimetría de corto plazo se testearon usando las hipótesis: $H_{0}$ : $\beta_{1, k}=\beta_{2, k}$ contra $H_{0}$ : $\beta_{1, k} \neq \beta_{2, k}$ y $H_{0}: \gamma_{1, k}^{B}=\gamma_{2, k}^{A}$ contra $H_{0}: \gamma_{1, k}^{B} \neq \gamma_{2, k}^{A}$ para ajuste de precios productor y mayorista, respectivamente. Finalmente, se analizó el impacto acumulativo que entregan al modelo los efectos positivos y negativos de la variación de precios: $H_{0}: \sum_{k=1}^{k} \beta_{1, k}=\sum_{k=1}^{k} \beta_{2, k}$ contra su respectiva $H_{A}$.

\section{B. Elasticidad precio-oferta}

Para obtener la elasticidad precio-oferta de los productores y generar una relación teórica con el poder de mercado mayorista, se siguió el marco 
propuesto por Sexton y Zhang (2001), Thille (2006) y ampliado por Assefa et al. (2017) para el caso de dos actores en la cadena productiva. Así, las funciones inversas de oferta y demanda, para productor y mayorista, vienen dadas por:

$$
\begin{gathered}
P_{p}=S\left(Q_{p}, Z_{p}\right) \\
P_{w}=D\left(Q_{w}, Z_{w}\right)
\end{gathered}
$$

donde $P_{p}$ y $P_{w}$ son los precios por kilo de carne a productor y mayorista, respectivamente; $Q_{p}$ y $Q_{w}$ las cantidades agregadas (ofrecidas y demandadas) de carne expresadas en kilos y $Z_{p}$ y $Z_{w}$ representan cambios exógenos de oferta y demanda.

Asumiendo costos de procesamiento y comercialización constantes (Sexton \& Zhang, 2001; Verreth et al., 2015), la función de beneficios de un mayorista $i$ es (omitiendo cambios exógenos de oferta y demanda):

$$
\pi_{w, i}=D\left(Q_{w}\right) q_{w, i}-S\left(Q_{p}\right) q_{w, i}-c_{w, i} q_{w, i},
$$

donde $q_{w, i}$ y $c_{w, i}$ corresponden a la producción y el costo unitario de comercialización y procesamiento del mayorista $i$. Agregando cantidades y costos, el problema de maximización de beneficios de un mayorista representativo sigue la siguiente relación de largo plazo entre precios mayorista y precios al productor ${ }^{3}$ :

$$
P_{w}\left(1-\frac{\phi_{w r}}{\varphi_{w}}\right)=P_{p}\left(1+\frac{\phi_{w p}}{\delta_{p}}\right)+c_{w}
$$

Siendo $\phi_{\mathrm{wr}}=\frac{\partial Q_{w}}{\partial q_{w}} \frac{q_{w}}{Q_{w}}$ una medida del poder de mercado de mayoristas hacia minoristas (retail), $\varphi_{w}=\frac{\partial Q_{w}}{\partial P_{w}} \frac{P_{w}}{Q_{w}}$ la elasticidad precio-demanda de mayoristas, $\phi_{w p}=\frac{\partial Q_{p}}{\partial q_{w}} \frac{q_{u c}}{Q_{p}}$ la elasticidad conjetural que mide el poder oligopsónico desde mayorista hacia productores y $\delta_{p}=\frac{\partial Q_{p}}{\partial P_{p}} \frac{P_{p}}{Q_{p}}$ la elasticidad precio-oferta de los productores. El valor esperado de $\varphi_{w p}$ va desde 0 , en caso de competencia perfecta, hasta 1 para el caso de monopolio. Valores intermedios, indican poder de oligopsonio en mayoristas.

3 Para detalles ver Sexton y Zhang (2001) y Verreth et al. (2015). 


\section{Datos}

Este estudio utiliza datos de series de tiempo mensuales desde enero 1982 a diciembre $2016^{4}$. Los precios al productor $\left(P_{p}\right)$ bovino y porcino corresponden a pesos por kilogramo $(\$ / \mathrm{kg})$ de carne bovina y cerdo de consumo; y los precios a mayorista $\left(P_{w}\right)$ son medidos en $\$ / \mathrm{kg}$ de carne de vacuno y carne de cerdo. Ambas series fueron obtenidas desde la Oficina de Estudios y Políticas Agrarias (ODEPA), dependiente del Ministerio de Agricultura de Chile. Para la estimación de la elasticidad conjetural tiempovariante, los precios de insumos: maíz (\$/kg USA Yellow No. 2 Golfo), trigo ( $\$ / \mathrm{kg}$, Hard Red Winter No. 2, FOB Golfo, USA); urea $(\$ / \mathrm{kg})$ y el precio de la leche ( $\$ / \ell$ a productor), provienen de ODEPA; el precio de la energía $^{5}$ (\$/kWh) desde la Comisión Nacional de Electricidad (CNE, 2019); índice general de remuneraciones desde el Banco Central de Chile (2019); las existencias de animales (miles de cabezas) ${ }^{6}$ y animales vendidos (toneladas de carne en vara) desde FAOSTAT (2019) y ODEPA (2019). Finalmente, todas las series de precios agrícolas fueron deflactadas según variación del Índice de Precios al Consumidor (IPC) de diciembre 2016. El precio medio de la energía eléctrica se actualizó a partir del valor de la Unidad de Fomento al 31 de diciembre de 2016 disponible en el sitio web del Servicio de Impuestos Internos de Chile (SII, 2019).

La Tabla 1 reporta estadística descriptiva de las principales variables usadas en la estimación del TECM especificado en (3). En general, la carne bovina presenta los niveles de precios más altos entre ambas industrias, alcanzando $\$ 932,06 / \mathrm{kg}$ y $\$ 2126,09 / \mathrm{kg}$ promedio para productor y mayorista, respectivamente. La elasticidad conjetural es mayor también en bovinos, promediando 0,626 versus 0,414 del sector porcino, haciendo suponer un mayor poder de mercado en la primera industria.

4 La serie utilizada, corresponde al horizonte temporal más amplio del que se tiene registro a la fecha del estudio.

5 Precio de nudo, Sistema Interconectado Central (SIC).

6 Datos disponibles en frecuencia anual. 
Troncoso-Sepúlveda: Poder de mercado y transmisión asimétrica de precios en Chile...

Tabla 1. Estadistica descriptiva precios al productor y mayorista

\begin{tabular}{lcccc}
\hline & Media $(\$ / \mathrm{kg})$ & Máximo $(\$ / \mathrm{kg})$ & Mínimo $(\$ / \mathrm{kg})$ & Desv. Est. \\
\hline Carne Bovina & & & & \\
Precios productor $\left(P_{p, t}\right)$ & 932,06 & 1462,16 & 584,82 & 204,63 \\
Precios mayorista $\left(P_{w, t}\right)$ & 2126,09 & 2992,49 & 1544,93 & 377,26 \\
Elasticidad conjetural $\left(\varphi_{w p, t}\right)$ & 0,626 & 0,641 & 0,611 & 0,009 \\
Carne Porcina & & & & \\
Precios productor $\left(P_{p, t}\right)$ & 905,44 & 1544,81 & 344,19 & 243,03 \\
Precios mayorista $\left(P_{w, t}\right)$ & 1494,65 & 2290,29 & 761,02 & 253,96 \\
Elasticidad conjetural $\left(\varphi_{w p, t}\right)$ & 0,414 & 0,569 & 0,259 & 0,09 \\
Insumos & & & & 17,778 \\
Salarios $\left(P_{s, t}\right)$ & 70,121 & 103,22 & 43,63 & 13,069 \\
\hline Precio electricidad $\left(P_{e, t}\right)$ & 48,34 & 80,58 & 25,79 & \\
\hline
\end{tabular}

Fuente: elaboración propia con datos de ODEPA (2019).

La Figura 1 presenta la evolución de los precios promedios mensuales de productores y mayoristas desde enero de 1982 a diciembre de 2016, según datos obtenidos desde ODEPA (2019):

En general, en cada mercado los precios a mayorista siguen cercanamente la tendencia de los precios al productor. En bovinos, por ejemplo, se aprecia un período de caída de precios entre 1992 y 1997 y una tendencia creciente a partir de 2004, atribuibles a variaciones de la producción de carne en vara nacional y que se transmiten hacia el sector mayorista. En porcinos, el efecto es similar, aunque la brecha de precios productor-mayorista es claramente menor. 
Figura 1. Evolución precios bovinos y porcinos 1982-2016
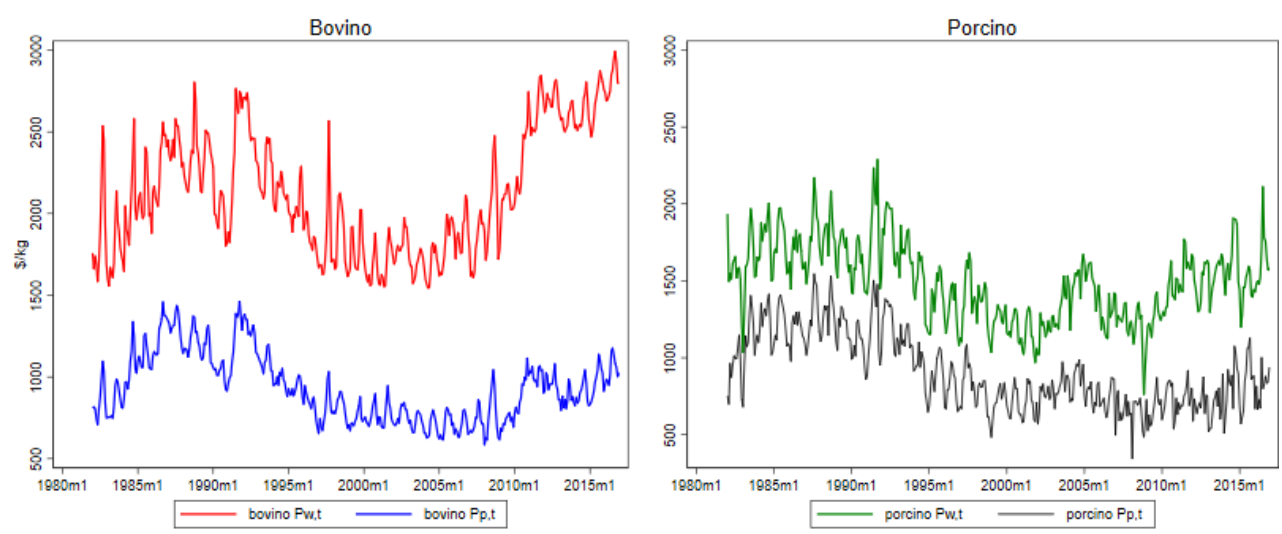

Fuente: elaboración propia con datos de ODEPA (2019).

\section{Resultados empíricos}

Previo a la estimación del modelo, se aplicaron las pruebas de raíz unitaria Augmented Dickey Fuller (ADF) y Kwiatkowski- Phillips-Schmidt-Shin (KPSS) a cada una de las variables utilizadas para estudiar su estacionariedad ${ }^{7}$. Como se puede apreciar en la Tabla 2, los resultados sugieren que todas las series son no estacionarias en niveles, pero I(1) en sus primeras diferencias al $1 \%$ de significancia estadística.

\section{A. Resultados modelos TAR/MTAR}

Las primeras tres filas de la Tabla 3, muestran los resultados de la prueba BBC que contrasta la hipótesis nula $\left(H_{0}\right)$ de raíz unitaria, contra la hipótesis alternativa $\left(H_{A}\right)$ de modelo estacionario con tres regímenes (Bec et al., 2004). La cuarta fila, muestra la prueba de Hansen para la $H_{0}$ de un régimen o linealidad en el proceso AR de la perturbación $\varepsilon_{t}$ versus la $H_{A}$ de tres regímenes o no linealidad. Ambas pruebas hacen inferencia directa sobre la validez de usar un modelo de cointegración por umbral con tres regímenes.

La especificación ADF, KPSS y resultados de la prueba de raíz unitaria Phillips-Perron, se encuentran disponibles previa solicitud al autor. 
Troncoso-Sepúlveda: Poder de mercado y transmisión asimétrica de precios en Chile...

Los resultados de las tres versiones de la prueba BBC (max-LR, LM y Wald) rechazan la $H_{0}$ al $1 \%$ de significancia para los precios bovinos y porcinos señalando cointegración por umbral en ambas series. El estadístico de Hansen (cuarta fila), arroja resultados favorables para la $H_{A}$ en el caso porcino, pero no permite rechazar la $H_{0}$ en bovinos. En general, ambas pruebas indican que las series de precios siguen un proceso TAR bajo la especificación propuesta con tres regímenes.

Tabla 2. Resultados test ADF y KPSS

\begin{tabular}{lcccccccc}
\hline & \multicolumn{2}{c}{ Productor } & \multicolumn{2}{c}{ Mayorista } & \multicolumn{2}{c}{ Salario } & \multicolumn{2}{c}{ Electricidad } \\
\cline { 2 - 9 } & $P_{p, t}$ & $\Delta P_{p, t}$ & $P w, t$ & $\Delta P_{w, t}$ & $P_{s, t}$ & $\Delta P_{s, t}$ & $P_{e, t}$ & $\Delta P_{e, t}$ \\
\hline Bovinos & & & & & & & & \\
ADF & $-0,39$ & $-12,45^{* * *}$ & $-0,12$ & $-16,33^{* * *}$ & 3,70 & $-10,73^{* * *}$ & $-0,15$ & $-11,05^{* * *}$ \\
KPSS & $3,52^{* * *}$ & 0,03 & $1,90^{* * *}$ & 0,03 & $10,40^{* * *}$ & 0,50 & $4,09^{* * *}$ & 0,13 \\
Porcinos & & & & & & & & \\
ADF & $-0,93$ & $-12,61^{* * *}$ & $-0,69$ & $-11,95^{* * *}$ & & & & \\
KPSS & $6,62^{* * *}$ & 0,02 & $3,11^{* * *}$ & 0,02 & & & & \\
\hline
\end{tabular}

Nota: ${ }^{* * *}$ señala rechazo de hipótesis nula al $1 \%$ de significancia. Salario y electricidad variables comunes en bovinos y porcinos.

Fuente: elaboración propia con datos de ODEPA (2019).

Las filas 5 y 6 de la Tabla 3 reportan el criterio de información AIC de los modelos TAR y MTAR para $\varepsilon_{t}$. El estadístico AIC favorece en ambos casos al MTAR indicando que las relaciones de precios bovinos y porcinos son mejor descritas usando la primera diferencia del ECT como variable umbral que la variable en nivel. En las tres filas siguientes, los coeficientes $\eta_{1}$ para los precios bovinos, son estadísticamente significativos en los tres regímenes y señalan una reducción del AR bajo la especificación MTAR con tres regímenes. Así, cuando $\Delta E C T_{t-1}$ está bajo su equilibrio de largo plazo, la velocidad de ajuste es $-0,506$ y cuando se encuentra por sobre el equilibrio es $-0,231$, considerablemente menor. Resultado similar se obtiene en la relación de precios porcinos, salvo que el coeficiente para el régimen bajo $\left(\eta_{1}^{B}\right)$ es 
estadísticamente no significativo. En la última fila, ambas hipótesis de ajuste simétrico en los regímenes bajo y alto, $\eta_{1}^{B}=\eta_{1}^{A}$, son rechazadas al $10 \%$ de significancia. En consecuencia, la velocidad de ajuste de precios bovinos y porcinos es significativamente diferente en los regímenes bajo y alto, es decir, el proceso de ajuste es más rápido en la fase creciente y más lento en la fase decreciente de precios ${ }^{8}$.

Tabla 3. Resultados modelos TAR y MTAR

\begin{tabular}{lcccc}
\hline \multirow{2}{*}{ Test/Parámetro } & \multicolumn{2}{c}{ Bovinos } & \multicolumn{2}{c}{ Porcinos } \\
\cline { 2 - 5 } & Est/Coef. & Valor-p & Est/Coef. & Valor-p \\
\hline Raíz unitaria vs TAR estacionario & $36,574^{* * *}$ & & $40,091^{* * *}$ & \\
BBC max-LR $^{a}$ & $35,019^{* * *}$ & & $38,219^{* * *}$ & \\
BBC max-L & & & $42,087^{* * *}$ & \\
BBC max-Wald $^{c}$ & $38,222^{* * *}$ & & $44,433^{* * *}$ & $<0,001$ \\
Test Hansen 1 vs 3 regímenes & 12,817 & 0,480 & -2224 & \\
TAR-AIC & -2562 & & -2225 & \\
MTAR-AIC & -2563 & & $-0,089$ & 0,324 \\
$\eta_{1}^{B}$ & $-0,506^{* * *}$ & $<0,001$ & & \\
$\eta_{1}^{M}$ & $-0,399^{* * *}$ & $<0,001$ & $-0,410^{* * *}$ & $<0,001$ \\
$\eta_{1}^{A}$ & $-0,234^{*}$ & 0,048 & $-0,293^{* * *}$ & $<0,001$ \\
$\eta_{1}^{B}=\eta_{1}^{A}$ & $3,559^{*}$ & 0,059 & $3,423^{*}$ & 0,064 \\
\hline
\end{tabular}

Nota: ${ }^{* * *},{ }^{* *}, \mathrm{y}^{*}$ denota significancia estadística al $1 \%, 5 \%$ y $10 \%$, respectivamente. ${ }^{a}$ Valores críticos: $15,772,17,898$ y 22,232 al $10 \%, 5 \%$ y $1 \%$ de significancia, respectivamente, ${ }^{b}$ Valores críticos: 15,587 , 17,630 y 21,756 al $10 \%, 5 \%$ y $1 \%$ de significancia, respectivamente, ${ }^{c}$ Valores críticos: $16,181,18,400$ y 23,010 al $10 \%, 5 \%$ y $1 \%$ de significancia, respectivamente.

Fuente: elaboración propia con datos de ODEPA (2019).

8 Efecto rockets and feathers. 
Troncoso-Sepúlveda: Poder de mercado y transmisión asimétrica de precios en Chile...

\section{B. Resultados TECM}

La Tabla 4 reporta el estadístico AIC y la estimación del modelo propuesto. El estadístico AIC favorece ampliamente al TECM y, por tanto, todos los resultados siguen esa estructura. Los parámetros $\theta_{0}^{B}, \theta_{0}^{M}$ y $\theta_{0}^{A}$, representan la velocidad de ajuste hacia el equilibrio de largo plazo en cada régimen cuando no se considera el poder de mercado como determinante de la tasa de ajuste de precios. En ambos modelos, $\operatorname{los} \theta_{0}^{B}$ estimados resultan estadísticamente no significativos, aunque en el caso porcino el signo y la condición de estacionariedad esperada se mantiene $\left(\theta_{0}^{B}<1\right)$. En los regímenes medio y alto, cuando $\tau_{1}<$ $\Delta E C T_{t-1} \leq \tau_{2}$ y $\Delta E C T_{t-1}>\tau_{2}$, los coeficientes $\theta_{0}$ son significativos, de magnitud y signo esperado y decrecientes en los dos modelos, es decir, cuando los precios se hallan en fase creciente (relativo a régimen alto) la velocidad de ajuste es considerablemente mayor que cuando están decreciendo. Concretamente, los precios mayoristas se ajustan a una tasa del 2,1\% (bovinos) y 3,2\% (porcinos) mensual hasta el equilibrio de largo plazo en el régimen medio, decayendo a 1,2\% y 2,6\% durante el régimen alto. Este resultado es consistente con los hallazgos desde el modelo MTAR presentados en la Tabla 3.

El efecto del poder de mercado $\left(M P_{t}\right)$ representado por $\theta_{1}^{i}$ en (4), (5) y (6) reporta el signo esperado en bovinos y porcinos. En el régimen bajo, cuando los precios se hallan por debajo del equilibrio, los mayoristas deberían ejercer su poder de mercado para acelerar el proceso de ajuste y mejorar bajos márgenes de ganancia, es decir, $\theta_{0}^{B}<0$. Sin embargo, en ambos modelos el poder de mercado es no significativo en el primer régimen. En los regímenes medio y alto, se espera que el poder de mercado mayorista ralentice el ajuste de precios a la baja para mantener márgenes altos y maximizar beneficios, es $\operatorname{decir,} \theta_{1}^{M}>0$ y $\theta_{1}^{A}>0$. En ambos casos, las estimaciones de $\theta_{1}^{M}$ y $\theta_{1}^{A}$ son positivos y numéricamente mayores $(33,199$ y 18,017 para bovinos y 1,012 y 0,617 para porcinos, respectivamente) a $\operatorname{los} \theta_{1}^{B}$, reflejando que el poder de mercado es más fuerte cuando ralentiza la disminución de precios que cuando acelera el aumento de precios mayoristas. No obstante, este efecto presenta significancia estadística sólo en el caso bovino. Estos resultados son consistentes con Acharya et al. (2011), Surathkal \& Chung (2019) y Liu et al. (2019). 
Tabla 4. Resultados TECM

\begin{tabular}{|c|c|c|c|c|c|c|}
\hline \multirow[b]{2}{*}{ AIC ECM } & \multicolumn{3}{|c|}{ Bovinos } & \multicolumn{3}{|c|}{ Porcinos } \\
\hline & \multicolumn{3}{|c|}{$-1475,802$} & \multicolumn{3}{|c|}{$-1146,679$} \\
\hline AIC TECM & \multicolumn{3}{|c|}{$-2677,536$} & \multicolumn{3}{|c|}{$-2336,265$} \\
\hline \multirow{2}{*}{ Variable/Régimen } & Bajo & Medio & Alto & Bajo & Medio & Alto \\
\hline & \multicolumn{3}{|c|}{ Coeficiente } & \multicolumn{3}{|c|}{ Coeficiente } \\
\hline \multirow{2}{*}{ const, $\gamma_{0}$} & \multicolumn{3}{|c|}{0,006} & \multicolumn{3}{|c|}{$0,274^{* *}$} \\
\hline & & $(0,097)$ & & & $(0,135)$ & \\
\hline \multirow{2}{*}{$E C T_{t-1}, \theta_{0}$} & 0,003 & $-0,021^{* *}$ & $-0,012^{*}$ & $-0,007$ & $-0,032^{* *}$ & $-0,026^{* *}$ \\
\hline & $(0,006)$ & $(0,010)$ & $(0,007)$ & $(0,016)$ & $(0,014)$ & $(0,012)$ \\
\hline \multirow{2}{*}{$E C T_{t-1} \times M P_{t}, \theta_{1}$} & $-6,114$ & $33,199^{* *}$ & $18,017^{* *}$ & $-0,519$ & 1,012 & 0,617 \\
\hline & $(4,937)$ & $(13,652)$ & $(7,977)$ & $(0,905)$ & $(0,679)$ & $(0,541)$ \\
\hline \multirow{2}{*}{$\Delta P_{w, t-1}, \gamma_{1,1}$} & $-0,019$ & $-0,612$ & $-0,213^{* *}$ & $-0,245^{* *}$ & $-0,275^{* *}$ & $-0,175^{* *}$ \\
\hline & $(0,072)$ & $(0,682)$ & $(0,105)$ & $(0,103)$ & $(0,127)$ & $(0,083)$ \\
\hline \multirow{2}{*}{$\Delta P_{w, t-2}, \gamma_{1,2}$} & $-0,148^{* * *}$ & $-0,355^{* * *}$ & $-0,138$ & $-0,457^{* * *}$ & $-0,218^{* * *}$ & $-0,069$ \\
\hline & $(0,049)$ & $(0,134)$ & $(0,100)$ & $(0,110)$ & $(0,080)$ & $(0,056)$ \\
\hline \multirow{2}{*}{$\Delta P_{p, t}^{+}, \beta_{1,0}$} & $0,264^{* * *}$ & $1,069^{* * *}$ & $0,480^{* * *}$ & $0,402^{* * *}$ & $0,618^{* * *}$ & $0,463^{* * *}$ \\
\hline & $(0,080)$ & $(0,207)$ & $(0,104)$ & $(0,068)$ & $(0,085)$ & $(0,074)$ \\
\hline \multirow{2}{*}{$\Delta P_{p, t-1}^{+}, \beta_{1,1}$} & 0,366 & 0,541 & $0,561^{* * *}$ & 1,714 & 0,118 & $0,128^{* *}$ \\
\hline & $(0,241)$ & $(0,582)$ & $(0,097)$ & $(2,699)$ & $(0,434)$ & $(0,054)$ \\
\hline \multirow{2}{*}{$\Delta P_{p, t-2}^{+}, \beta_{1,2}$} & 0,149 & 0,315 & 0,064 & $0,276^{* *}$ & $0,244^{* * *}$ & 0,015 \\
\hline & $(0,099)$ & $(0,217)$ & $(0,117)$ & $(0,110)$ & $(0,079)$ & $(0,054)$ \\
\hline \multirow{2}{*}{$\Delta P_{p, t}^{-}, \beta_{2,0}$} & $0,393^{* * *}$ & $-0,154$ & $0,202^{*}$ & $0,696^{* * *}$ & $0,398^{* * *}$ & $0,117^{* * *}$ \\
\hline & $(0,074)$ & $(0,270)$ & $(0,118)$ & $(0,098)$ & $(0,074)$ & $(0,045)$ \\
\hline \multirow{2}{*}{$\Delta P_{p, t-1}^{-}, \beta_{2,1}$} & $0,369^{* * *}$ & $3,150^{* * *}$ & $-0,786$ & $0,647^{* * *}$ & $0,663^{* * *}$ & $3,654^{*}$ \\
\hline & $(0,073)$ & $(1,024)$ & $(0,797)$ & $(0,075)$ & $(0,161)$ & $(2,042)$ \\
\hline \multirow{2}{*}{$\Delta P_{p, t-2}^{-}, \beta_{2,2}$} & $0,146^{* *}$ & 0,121 & 0,369 & $0,331^{* * *}$ & 0,063 & 0,046 \\
\hline & $(0,075)$ & $(0,281)$ & $(0,233)$ & $(0,128)$ & $(0,107)$ & $(0,055)$ \\
\hline \multirow{2}{*}{$\Delta P_{s}, \gamma_{2}$} & 0,263 & 0,097 & 0,029 & $-0,647$ & 0,411 & 0,510 \\
\hline & $(0,277)$ & $(0,791)$ & $(0,414)$ & $(0,680)$ & $(0,494)$ & $(0,414)$ \\
\hline \multirow{2}{*}{$\Delta P_{e}, \gamma_{3}$} & $-0,074$ & $0,686^{* * *}$ & $0,157^{*}$ & 0,001 & $-0,141$ & 0,010 \\
\hline & $(0,066)$ & $(0,225)$ & $(0,085)$ & $(0,267)$ & $(0,117)$ & $(0,096)$ \\
\hline
\end{tabular}

Nota: ${ }^{* *},{ }^{* *}, \mathrm{y}^{*}$ denota significancia estadística al $1 \%, 5 \%$ y $10 \%$, respectivamente. Errores estándar en paréntesis.

Fuente: elaboración propia a partir de estimaciones TECM usando datos de ODEPA (2019). 
Las asimetrías de corto plazo son definidas como la diferencia en la respuesta de los precios mayoristas a aumentos o disminuciones de los precios al productor. El coeficiente de cambio contemporáneo positivo $\beta_{1,0}$ es siempre significativo y muestra valores más grandes en el régimen alto que en el bajo, lo cual indica que los mayoristas (bovinos y porcinos) responden más prontamente en el régimen alto ante variaciones positivas de los precios al productor. Los $\beta_{1,1}$ estimados son significativos sólo en el régimen alto y señalan velocidad de ajuste mayor en bovinos que porcinos $(0,561$ y 0,128 , respectivamente). Los coeficientes de variación precio-negativo; $\beta_{2,0}, \beta_{2,1} \mathrm{y}$ $\beta_{2,2}$; reflejan que el proceso de ajuste es más lento ante variaciones rezagadas que contemporáneas en el régimen bajo de cada modelo, en tanto se aprecian resultados mixtos durante los regímenes medio y alto.

El ajuste de precios de largo plazo se puede estimar mediante la suma de los coeficientes $\beta_{1, k}$ y $\beta_{2, k}$ para todos los rezagos. Así, el ajuste de largo plazo de mayoristas en respuesta a variaciones positivas de los precios al productor es $\sum_{k=0}^{2} \beta_{1, k}^{B}=0,779$ y 2,392 y $\sum_{k=0}^{2} \beta_{1, k}^{A}=1,105$ y 0,606 para bovinos y porcinos en regímenes bajo y alto, respectivamente. Estos resultados sugieren efectos opuestos en ambos mercados. Cuando los precios a mayorista bovino/porcino decrecen/crecen la transmisión de precios desde productor es más rápida que cuando los precios se encuentran creciendo/decreciendo. El ajuste ante cambios negativos advierte un efecto de transmisión contrario al de las variaciones positivas con $\sum_{k=0}^{2} \beta_{2, k}^{B}=0,908$ y 1,674 y $\sum_{k=0}^{2} \beta_{2, k}^{A}=$ $-0,215$ y 3,817 para bovinos y porcinos, en cada régimen. Ambos casos son consistentes con el fenómeno de 'rockets and feathers' hallado en la literatura sobre transmisión de precios.

\section{Test de asimetrías de corto/largo plazo}

La Tabla 5 presenta los resultados de las pruebas de hipótesis sobre el comportamiento de ajuste de precios de mayoristas, a partir de los coeficientes de la Tabla 4. El ajuste asimétrico general fue testeado comparando los $\Theta^{B}$ y $\Theta^{A 9}$. La diferencia de coeficientes sugiere que el ajuste de precios bovinos

$9 \quad$ Evaluados en la media de $M P_{t}$. 
es más rápido en el régimen bajo que en el alto, mientras que los precios a mayorista porcino presentan el efecto opuesto, es decir, mayor velocidad de ajuste en el régimen alto. Sin embargo, la prueba de hipótesis sobre la diferencia $\Theta^{B}-\Theta^{A}$ muestra que la $H_{0}$ de simetría general de largo plazo, sólo es rechazada en el caso porcino. Cuando el poder de mercado es considerado explícitamente, las $H_{0}: \theta_{0}^{B}=\theta_{0}^{A}$ y $H_{0}: \theta_{1}^{B}=\theta_{1}^{A}$ son rechazadas al $1 \%$ en bovinos y no rechazadas en porcinos.

Tabla 5. Resultados test asimetrías corto/largo plazo

\begin{tabular}{lcccc}
\hline \multirow{2}{*}{$H_{0}$} & \multicolumn{2}{c}{ Bovinos } & \multicolumn{2}{c}{ Porcinos } \\
\cline { 2 - 5 } & Diferencia & Valor-p & Diferencia & Valor-p \\
\hline$\Theta_{B}=\Theta_{A}$ & $-0,001$ & 0,855 & $0,004^{* * *}$ & 0,002 \\
$\theta_{0}^{B}=\theta_{0}^{A}$ & $0,014^{* * *}$ & 0,008 & 0,019 & 0,159 \\
$\theta_{1}^{B}=\theta_{1}^{A}$ & $-24,130^{* * *}$ & 0,009 & $-1,136$ & 0,276 \\
Asimétrica de corto/largo plazo & & & & \\
$\beta_{1,0}^{B}=\beta_{1,0}^{A}$ & $-0,264^{*}$ & 0,092 & $-0,060$ & 0,544 \\
$\beta_{1,1}^{B}=\beta_{1,1}^{A}$ & $-0,195$ & 0,452 & 1,585 & 0,557 \\
$\beta_{1,2}^{B}=\beta_{1,2}^{A}$ & 0,086 & 0,575 & $0,260^{* *}$ & 0,034 \\
$\beta_{1, k}^{B}=\sum_{k=0}^{2} \beta_{1, k}^{A}$ & $-0,325$ & 0,315 & 1,785 & 0.511 \\
$\beta_{2,0}^{B}=\beta_{2,0}^{A}$ & 0,190 & 0,173 & $0,579^{* * *}$ & 0,000 \\
$\beta_{2,1}^{B}=\beta_{2,1}^{A}$ & 1,155 & 0,149 & $-3,006$ & 0,141 \\
$\beta_{2,2}^{B}=\beta_{2,2}^{A}$ & $-0,223$ & 0,362 & $0,285^{* *}$ & 0,041 \\
$\sum_{k=0}^{2} \beta_{2, k}^{B}=\sum_{k=0}^{2} \beta_{2, k}^{A}$ & 1.122 & 0,189 & $-2,143$ & 0,294 \\
Asimetría intra-régimen & & & & \\
$\beta_{1,0}^{B}=\beta_{2,0}^{B}$ & $-0,129$ & 0,313 & $-0,294^{* *}$ & 0,027 \\
$\beta_{1,1}^{B}=\beta_{2,1}^{B}$ & $-0,004$ & 0,990 & 1,067 & 0,693 \\
$\beta_{1,2}^{B}=\beta_{2,2}^{B}$ & 0,003 & 0,982 & $-0,055$ & 0,746 \\
$\beta_{1, k}^{B}=\sum_{k=0}^{2} \beta_{2, k}^{B}$ & $-0,129$ & 0,691 & 0,718 & 0,788 \\
$\beta_{1,0}^{A}=\beta_{2,0}^{A}$ & 0,278 & 0,146 & $0,346^{* * *}$ & 0,001 \\
$\beta_{1,1}^{A}=\beta_{2,1}^{A}$ & $1,347^{*}$ & 0,100 & $-3,525^{*}$ & 0,086 \\
$\beta_{1,2}^{A}=\beta_{2,2}^{A}$ & $-0,306$ & 0,294 & $-0,030$ & 0,715 \\
$\beta_{1, k}^{A}=\sum_{k=0}^{2} \beta_{2, k}^{A}$ & 1,318 & 0,146 & $-3,210$ & 0,116 \\
\hline
\end{tabular}

Nota: ${ }^{* * *},{ }^{* *}, \mathrm{y}^{*}$ denota significancia estadística al $1 \%, 5 \%$ y $10 \%$, respectivamente.

Fuente: elaboración propia con datos de ODEPA (2019). 
Troncoso-Sepúlveda: Poder de mercado y transmisión asimétrica de precios en Chile...

Estos resultados permiten concluir que existen diferencias en el efecto del poder de mercado en los regímenes bajo y alto en bovinos y que dicho efecto es más acentuado cuando los precios se hallan en fase decreciente, es decir, existen asimetrías.

En cuanto a las hipótesis de simetría de corto y largo plazo, $H_{0}$ no es rechazada en casi todos los casos, a excepción de $H_{0}: \beta_{1,0}^{B}=\beta_{1,0}^{A}$ y $H_{0}: \beta_{1,1}^{A}=$ $\beta_{2,1}^{A}$ para bovinos, reportándose un comportamiento mucho más asimétrico en porcinos, tanto inter como intra-régimen. Por ejemplo, $H_{0}: \beta_{2,0}^{B}=\beta_{2,0}^{A}$ se rechaza al $1 \%$ de significancia reflejando la existencia de transmisión asimétrica desde productores a mayoristas cuando los primeros experimentan variaciones contemporáneas negativas de precios en los regímenes bajo y alto; y el rechazo de $H_{0}: \beta_{1,0}^{B}=\beta_{2,0}^{B}$ al $5 \%$ y $H_{0}: \beta_{1,0}^{A}=\beta_{2,0}^{A}$ al $1 \%$ insinúa que los precios a mayorista porcino reaccionan de forma distinta a cambios contemporáneos positivos o negativos de los precios al productor dentro de un régimen en particular.

\section{Resultados elasticidad precio-oferta}

Para obtener la elasticidad precio-oferta de productores, se estimó la relación de equilibrio descrita en la ecuación 1 por el método de Johansen ${ }^{10}$ y se utilizó la ecuación 10 para calcular el coeficiente en cada mercado. En la Tabla 6 se reportan algunos de estos resultados.

Cada relación de equilibrio de largo plazo, o vector de cointegración, puede ser estimada sin normalizar con respecto a los precios mayoristas. Así, los vectores para bovinos y porcinos se pueden escribir como: $0,360 P_{w, t-1}=$ $0,291 P_{p, t-1}+0,141 P_{s, t-1}+0,009 P_{e, t-1}-0,017$ y $0,021 P_{w, t-1}=$ $0,024 P_{p, t-1}+0,012 P_{s, t-1}+0,001 P_{e, t-1}-0,064$, respectivamente. Ya que $\left(1+\frac{\phi_{w p}}{\delta_{p}}\right) \neq 1(0,291$ y 0,024$)$, esto sugiere la presencia de poder oligopsónico desde mayoristas hacia productores en los mercados de carne bovina y porcina. Utilizando este resultado y la elasticidad conjetural promedio, se estimaron las elasticidades precio-oferta de productores en $\delta_{p}=0,883$ y 0,424 para bovinos y porcinos, respectivamente. Estos coeficientes señalan que, en

$\overline{10}$ El número de rezagos utilizados fue dos, según criterio HQIC. 
ambas industrias, la oferta de los productores reacciona de manera inelástica a cambios en el nivel de precios.

Tabla 6. Relación de equilibrio de largo plazo

\begin{tabular}{ccccc}
\hline \multirow{2}{*}{ Variable } & \multicolumn{2}{c}{ Bovinos } & \multicolumn{2}{c}{ Porcinos } \\
\cline { 2 - 5 } & Coeficiente & Valor-p & Coeficiente & Valor-p \\
\hline$P_{w, t-1}$ & 1 & - & 1 & - \\
$P_{p, t-1}$ & $-0,807^{* * *}$ & $<0,001$ & $-1,163^{* * *}$ & $<0,001$ \\
& $(0,032)$ & & $(0,068)$ & \\
$P_{s, t-1}$ & $-0,391^{* * *}$ & $<0,001$ & $-0,585^{* * *}$ & $<0,001$ \\
& $(0,027)$ & & $(0,072)$ & \\
$P_{e, t-1}$ & $-0,142^{* * *}$ & $<0,001$ & $-0,022$ & 0,621 \\
& $(0,022)$ & & $(0,044)$ & \\
const & 0,048 & - & 3,134 & - \\
\hline
\end{tabular}

Nota: ${ }^{* *},{ }^{* *}, \mathrm{y}^{*}$ denota significancia estadística al $1 \%, 5 \%$ y $10 \%$, respectivamente. Errores estándar en paréntesis.

Fuente: elaboración propia con datos de ODEPA (2019).

\section{Conclusiones}

El principal propósito de este artículo fue examinar el efecto del poder de mercado sobre el proceso de ajuste de precios productor-mayorista, en la industria de carne bovina y porcina chilena. Para ello, se utilizaron las elasticidades conjeturales como variable proxy del poder de mercado y se estimó un TECM con tres regímenes para distinguir el ajuste de precios según su comportamiento, creciente o decreciente con relación al equilibrio de largo plazo, y la transmisión desde productores.

Los resultados muestran, un efecto estadísticamente significativo del poder de mercado en el comportamiento de ajuste de precios bovinos. Cuando los precios se hallan en la fase decreciente, los mayoristas bovinos ejercen su poder de mercado para ralentizar el ajuste de precios a la baja y conservar márgenes de ganancia más altos durante más tiempo. Esto último, no se observó en el modelo porcino porque no fue posible probar que el 
coeficiente de velocidad de ajuste de largo plazo fuera función del poder de mercado. El ajuste asimétrico de precios también fue testeado sin considerar el efecto del poder de mercado en cada modelo. Así, cuando no se incluyó el poder de mercado, la hipótesis de simetría sólo fue rechazada en el caso porcino reforzando los resultados desde el TECM y permitiendo concluir que el poder de mercado mayorista genera asimetrías régimen-dependientes sólo en el sector bovino.

En cuanto a la transmisión de precios productor-mayorista, las asimetrías positivas de corto plazo señalaron que los mayoristas bovinos y porcinos reaccionan más rápidamente en el régimen alto que en el bajo, ante variaciones contemporáneas de los precios al productor. Por su parte, las asimetrías negativas reflejaron que el proceso de ajuste tiende a ser más veloz en respuesta a variaciones contemporáneas que rezagadas, en el régimen bajo de cada modelo. En el largo plazo, los coeficientes estimados sugieren efectos de transmisión opuestos ante variaciones de precios positivas y negativas en cada mercado, pero consistentes con el fenómeno 'rockets and feathers'. Las pruebas de hipótesis inter e intra-regímenes mostraron mayor evidencia de transmisión asimétrica de precios en porcinos que en bovinos. Finalmente, la estimación de elasticidad precio indica que la oferta de los productores reacciona menos que proporcionalmente a cambios en el nivel de precios.

Las implicancias de esta investigación pueden ser potencialmente útiles para hacedores de política o instituciones gubernamentales interesadas en regular la concentración y reducir consecuencias económicas como baja competencia, elevadas barreras de entrada, prácticas empresariales ilegales y perjuicios en precio, calidad y bienestar para los consumidores. Este artículo contribuye al amplio cuerpo de literatura existente, entregando ideas sobre cómo opera el poder de mercado en la transmisión de precios en un país en desarrollo como Chile, e indicando la importancia de considerar la estructura de mercado en la estimación de modelos de transmisión de precios y mejorar el rendimiento de aproximaciones basadas en cointegración por umbral.

Futuras investigaciones podrían utilizar nuevas medidas de poder de mercado para ser incorporadas en el análisis de transmisión de precios y sus determinantes, ya que algunos autores han mostrado que la estimación de 
elasticidades conjeturales puede ser sensible a especificación del modelo y forma funcional (Chung et al., 2018; Surathkal \& Chung, 2019). Además, en el caso chileno, existen diversas áreas hacia dónde dirigir futuras investigaciones. Una de ellas podría ser ampliar este tipo de trabajos a otros mercados como los cereales, forestal, productos marinos o mineros, para estudiar teórica y empíricamente el poder de mercado en las cadenas de valor. Otra dirección de futuros estudios sería comprender cómo impacta la competitividad de mercado a nivel bienestar de los consumidores en industrias más y menos competitivas. Finalmente, puede ser relevante explorar otros factores que podrían causar transmisión asimétrica de precios. Uno de ellos son los costos de ajuste, debido a que es más complejo para las empresas incrementar la producción cuando el costo de insumos va a la baja que disminuirla cuando el costo sube, porque en el primer caso se requiere contratación o compra de insumos. Además, las intervenciones de política podrían causar transmisión asimétrica de precios aun en mercados relativamente más competitivos, como señalan Cavicchioli, D. (2018) y Yu y Gould (2019), entre otros. Sin embargo, un desafío adicional es obtener datos relevantes para analizar el impacto de tales variables sobre la transmisión y el bienestar de los consumidores.

\section{Anexo}

\section{Resumen modelo de ecuaciones simultáneas para estimar $\varphi_{w p, t}$}

Para generar la elasticidad conjetural, se usó el marco propuesto por Muth \& Wohlgenant (1999) y adaptado por Vásquez Panizza (2014) para el mercado ganadero chileno y que se basa en la estimación 3SLS de un sistema de demanda y oferta como el siguiente:

\section{Bovinos:}

$$
\begin{gathered}
P_{p}=-\phi_{w p}\left[\frac{1}{\psi_{1}}\right] \frac{Q}{I}+\alpha_{1} Q+\alpha_{2} P_{s}+\alpha_{3} P_{e}+\alpha_{4} P_{w} \\
\frac{Q}{I}=\psi_{0}+\psi_{1} P_{p}+\psi_{2} P_{u}+\psi_{3} P_{m}+\psi_{4} P_{l}+\psi_{5} Q_{12}+\psi_{6} T
\end{gathered}
$$


Troncoso-Sepúlveda: Poder de mercado y transmisión asimétrica de precios en Chile...

Porcinos:

$$
\begin{aligned}
& P_{p}=-\phi_{w p}\left[\frac{1}{\psi_{1}}\right] \frac{Q}{I}+\alpha_{1} Q+\alpha_{2} P_{s}+\alpha_{3} P_{e}+\alpha_{4} P_{w} \\
& \frac{Q}{I}=\psi_{0}+\psi_{1} P_{p}+\psi_{2} P_{t}+\psi_{3} P_{m}+\psi_{4} Q_{12}+\psi_{5} T
\end{aligned}
$$

donde $P_{p}$ y $P_{w}$ son los precios al productor y mayorista; $P_{u}, P_{m}$ y $P_{t}$ los precios de insumos especializados: urea, maíz y trigo, respectivamente; $P_{l}$ el precio de la leche; $P_{s}$ y $P_{e}$ los salarios y el precio de la electricidad. $Q$ y $I$, la cantidad vendida y los inventarios según especie. Los coeficientes $\alpha$ 's y $\psi$ 's son parámetros a estimar y $\varphi_{w p}=\varphi_{0}+\varphi_{1} T$ la elasticidad conjetural, principal output de interés del sistema.

\section{Referencias}

Acharya, R. N., Kinnucan, H. W., \& Caudill, S. B. (2011). Asymmetric farm-retail price transmission and market power: A new test. Applied Economics 43(30), 4759-4768. https://doi.org/10.1080/00036846.2 010.498355

Alfaro, D., \& Olivera, M. (2009) Transmisión de precios y poder de mercado: el caso del ganado vacuno para faena. Ministerio de Economía y Finanzas de Uruguay. https://www.mef.gub.uy/innovaportal/file/1 414/1/20100729_informe_final_mercado_ganado_vacuno_faena.pdf

ASPROCER (junio de 2019). Análisis sectorial. Asociación Gremial de Productores de Cerdos de Chile. http://www.asprocer.cl/industria/ analisis-sectorial/

Assefa, T. T., Kuiper, W. E., \& Meuwissen, M. P. (2014). The effect of farmer market power on the degree of farm retail price transmission: A simulation model with an application to the dutch ware potato supply Chain. Agribusiness 30(4),424-437. https://doi.org/10.1002/agr.21371

Assefa, T. T., Meuwissen, M. P., Gardebroek, C., \& Oude Lansink, A. G. (2017). Price and Volatility Transmission and Market Power in the 
German Fresh Pork Supply Chain. Journal of Agricultural Economics 68(3), 861-880. https://doi.org/10.1111/1477-9552.12220

Banco Central de Chile (junio de 2019). Mercado Laboral y Demografía. https://si3.bcentral.cl/Siete/ES/Siete/Cuadro/CAP_EMP_REM _DEM/MN_EMP_REM_DEM13/ED_IND_REM_M_2016/c3a

Bec, F., Ben Salem, M., \& Carrasco, M. (2004). Tests for unit-root versus threshold specification with an application to the purchasing power parity relationship. Journal of Business and Economic Statistics 22(4), 382395. https://doi.org/10.1198/073500104000000389

Blake, N. S. \& Fomby, T. B. (1997). Threshold Cointegration. International Economic Review 38(3), 627. https://doi.org/10.2307/2527284

Brosig, S., Glauben, T., Götz, L., Weitzel, E. B., \& Bayaner, A. (2011). The Turkish wheat market: Spatial price transmission and the impact of transaction costs. Agribusiness 27(2), 147-161. https://doi.org/10.1 002/agr.20257

Bunte, F. \& Peerlings, J. (2003). Asymmetric price transmission due to market power in the case of supply shocks. Agribusiness, 19(1) 19-28. https: //doi.org/10.1002/agr.10040

Cavicchioli, D. (2018). Detecting market power along food supply chains: Evidence and methodological insights from the fluid milk sector in Italy. Agriculture, 8(12), 191. https://doi.org/10.3390/agriculture8120191

Ceballos, P. (2014). Transmisión de precios en el mercado de la carne en Chile [tesis de maestría, Universidad del Bío-Bío]. Repositorio Universidad del Bío-Bío. http://repobib.ubiobio.cl/jspui/bitstream/123456789/3 40/3/Ceballos\%20Garrido\%2C\%20Paulina\%20Andrea.pdf

Chan, K. S. (1993). Consistency and Limiting Distribution of the Least Squares Estimator of a Threshold Autoregressive Model. The Annals of Statistics 21(1), 520-533. https://doi.org/10.1214/aos/1176349040

Chavas, J.-P. \& Pan, F. (2019). The Dynamics and Volatility of Prices in a Vertical Sector. American Journal of Agricultural Economics 102(1), 353369. https://doi.org/10.1093/ajae/aaz038 
Troncoso-Sepúlveda: Poder de mercado y transmisión asimétrica de precios en Chile...

Chung, C., Park, S., and Lee, J. (2018). Estimating bilateral market power of processors and retailers in the U.S. beef industry. Agribusiness 34(4), 771-792. https://doi.org/10.1002/agr.21559

CNE (junio de 2019). Estadísticas. Comisión Nacional de Energía. https: //www.cne.cl/estadisticas/electricidad/

Cudjoe, G., Breisinger, C., \& Diao, X. (2010). Local impacts of a global crisis: Food price transmission, consumer welfare and poverty in Ghana. Food Policy 35(4), 294-302. https://doi.org/10.1016/j.foodpol.2010.01.004

Enders, W. \& Siklos, P. L. (2001). Cointegration and threshold adjustment. Journal of Business and Economic Statistics 19(2), 166-176. https://doi. org/10.1198/073500101316970395

Engle, R. F. \& Granger, C. W. J. (1987). Co-Integration and Error Correction: Representation, Estimation, and Testing. Econometrica 55(2), 251-276. https://doi.org/10.2307/1913236

FAO. (2019). Carne y Productos Cárnicos. Producción y Sanidad Animal. http://www.fao.org/ag/againfo/themes/es/meat/home.html

FAO. (2018). Perspectivas alimentarias - Resumen de Mercado- http://www. fao.org/3/CA0910ES/ca0910es.pdf

FAOSTAT (junio de 2019). Animales Vivos. Food and Agriculture Organization of the United Nations. http://www.fao.org/faostat/en/\#data/QA

Farías, C., Espinoza, H., \& Giacomozzi, J. (2016). Transmisión de precios en la cadena de carne bovina en Chile. Oficina de Estudios y Políticas Agrarias. https://www.odepa.gob.cl/wp-content/uploads/2017/12/ca denaCarneBovina.pdf

Farrell, M. J. (1952). Irreversible Demand Functions. Econometrica 20(2), 171-186. https://doi.org/0012-9682(195204)20:2<171:IDF> 2.0.CO;2-Z

Frey, G. y Manera, M. (2007). Econometric models of asymmetric price transmission. Journal of Economic Surveys 21(2), 349-415. https://doi. org/10.1111/j.1467-6419.2007.00507.x 
Geweke, J. (2004). Issues in the Rockets and Feathers Gasoline Price Literature. Technical report.

Götz, L., Glauben, T., \& Brümmer, B. (2013). Wheat export restrictions and domestic market effects in Russia and Ukraine during the food crisis. Food Policy 38 (1), 214-226. https//doi.org.10.1016/j.foodpol.2012. 12.001

Houck, J. P. (1977). An Approach to Specifying and Estimating Nonreversible Functions. American Journal of Agricultural Economics 59(3), 570-572. https://doi.org/10.2307/1239663

Lewis, M. S. (2011). Asymmetric Price Adjustment and Consumer Search: An Examination of the Retail Gasoline Market. Journal of Economics and Management Strategy, 20(2), 409-449. https://doi.org/10.1111/j. 1530-9134.2011.00293.x

Liu, Y., Chen, X., \& Rabinowitz, A. N. (2019). The role of retail market power and state regulations in the heterogeneity of farm-retail price transmission of private label and branded products. Agricultural Economics 50(1), 91-99. https://doi.org/10.1111/agec.12468

McCorriston, S. (2002). Why should imperfect competition matter to agricultural economists? European Review of Agriculture Economics, 29(3),349-371. https://doi.org/10.1093/eurrag/29.3.349

Meyer, J. (2004). Measuring market integration in the presence of transaction costs - A threshold vector error correction approach. Agricultural Economics 31 (2-3), 327-334. https://doi.org/10.1016/j.agecon.2 004.09.017

Meyer, J. \& von Cramon-Taubadel, S. (2005). Asymmetric price transmission: A survey. Journal of Agricultural Economics, 55 (3), 581-611. https: //doi.org/10.1111/j.1477-9552.2004.tb00116.x

Miller, D. J. \& Hayenga, M. L. (2001). Price cycles and asymmetric price transmission in the U.S. pork market. American Journal of Agricultural Economics 83(3), 551-562. https://doi.org/10.1111/0002-9092.00177 
Troncoso-Sepúlveda: Poder de mercado y transmisión asimétrica de precios en Chile...

Muth, M. K. \& Wohlgenant, M. K. (1999). Measuring the Degree of Oligopsony Power in the Beef Packing Industry in the Absence of Marketing Input Quantity Data. Journal of Agricultural and Resource Economics 24(2), 299-312. https://www.jstor.org/stable/40987024

ODEPA (junio 2019). Series de tiempo. Oficina de Estudios y Políticas Agrarias. https:/www.odepa.gob.cl/precios/series-de-tiempo

Vásquez Panizza, R. A. (2014). Market power estimation in the Chilean cattle market [Tesis de maestría, Universidad de Purdue]. https://docs.lib.pur due.edu/open_access_theses/393/

Radchenko, S. (2005). Lags in the response of gasoline prices to changes in crude oil prices: The role of short-term and long-term shocks. Energy Economics 27(4), 573-602. https://doi.org/10.1016/j.eneco.2005.04. 004

Richards, T. J., Gomez, M. I., \& Lee, J. (2014). Pass-through and consumer search: An empirical analysis. American Journal of Agricultural Economics 96(4), 1049-1069. https://doi.org/10.1093/ajae/aau009

Sexton, R. J. \& Zhang, M. (2001). An assessment of the impact of food industry market power on U.S. consumers food industry market power and U.S. consumers. Agribusiness 17(1), 59-79. https://doi.org/10.100 2/1520-6297(200124)17:1<59::AID-AGR1003>3.0.CO;2-D

SII (junio de 2019). Unidad de Fomento. Servicio de Impuestos Internos. https://www.sii.cl/valores_y_fechas/uf/uf2021.htm

Surathkal, P. \& Chung, C. (2019). Effects of packers' inventory and market power on price adjustments in the U.S. beef industry. Applied Economics 51(46), 5076-5089. https://doi.org/10.1080/00036846.2019.161070 8

Tansuchat, R., Maneejuk, P., Wiboonpongse, A., \& Sriboonchitta, S. (2016). Price transmission mechanism in the thai rice market. Studies in Computational Intelligence 622, 451-461. https://doi.org/10.1007/ 978-3-319-27284-9_29 
Thille, H. (2006). Inventories, market structure, and price volatility. Journal of Economic Dynamics and Control 30(7), 1081-1104. https://doi.org/ 10.1016/j.jedc.2005.04.003

Tong, H. (1990). Non-linear time series: a dynamical system approach. Oxford University Press. https://doi.org/10.1137/1034036

Troncoso-Sepúlveda, R. (2019). Transmisión de los precios del arroz en Colombia y el mundo. Lecturas de Economía 91, 151-179. https://doi. org/10.17533/udea.le.n91a05

Vargas, G. \& Raddatz, W. F. M. (2004). Divergencia en la organización de la industria cárnica: un análisis comparativo de Estados Unidos y Chile. Ciencia e Investigación Agraria 31 (2),75-90. http://dx.doi.org/10.7764 /rcia.v31i2.284

Verreth, D. M., Emvalomatis, G., Bunte, F., Kemp, R., \& Oude Lansink, A. G. (2015). Price Transmission, International Trade, and Asymmetric Relationships in the Dutch Agri-Food Chain. Agribusiness 31(4), 521 542. https://doi.org/10.1002/agr.21420

Wolffram, R. (1971). Positivistic Measures of Aggregate Supply Elasticities: Some New Approaches: Some Critical Notes. American Journal of Agricultural Economics 53(2), 356. https://doi.org/10.2307/1237462

Yu, C. \& Gould, B. W. (2019). Market power and farm-retail price transmission: The case of US fluid milk markets. Agribusiness 35 (4), 537-555. https://doi.org/10.1002/agr.21606 